\title{
A Study of Brewitt-Taylor's Translation of San Guo Yan $Y i$ from the Perspective of Polysystem Theory
}

\author{
Liu Silang ${ }^{1, a^{*}}$, Li Xiangmin ${ }^{2, b}$
}

\author{
${ }^{1}$ School of English Language, Literature, and Culture, Beijing International Studies University, Beijing, China \\ ${ }^{2}$ School of English Language, Literature, and Culture, Beijing International Studies University, Beijing, China \\ $a^{*}$ lauszelong@foxmail.com \\ blixm@bisu.edu.cn
}

\begin{abstract}
San Guo Yan $Y i$ is the first Chinese historical novel divided into chapters. Its English translation primarily includes two editions translated by two foreign scholars, Moss Roberts, and C.H. Brewitt-Taylor, and one edition translated by Chinese scholar, Yu Sumei. For the time being, the research among the China's Academia on the English editions of San Guo Yan Yi is mainly based on translation theories, mostly in praise of Roberts' edition rather than Brewitt-Taylor's version, while there has been little research on Brewitt-Taylor's edition from the perspective of Polysystem Theory. Polysystem Theory has enriched translation research methods by means of multi-systems exemplified by the cultures beyond the texts. From the perspective of Polysystem Theory, this research took Brewitt-Taylor's translation of San Guo Yan Yi as an example to examine how the status of Chinese and English literature could determine the translation intentions. Besides, this research illustrated with characteristic examples the influence of various elements in the novel on the translation behaviors and the diction strategies, and pinpointed the shortcomings of Polysystem Theory presented in Brewitt-Taylor's translation.
\end{abstract}

Keywords: Polysystem Theory, Brewitt-Taylor's translation of San Guo Yan Yi, translation of the Chinese Classics, translation intentions

\section{多元系统论视角下的《三国演义》邓罗译本研究}

刘思朗 ${ }^{1, a *}$ ，李向民 ${ }^{2, b}$

1 北京第二外国语学院英语学院, 北京, 中国

2 北京第二外国语学院英语学院, 北京, 中国

a*lauszelong@foxmail.com

${ }^{b}$ lixm@bisu.edu.cn

\section{摘要}

《三国演义》是我国首部章回体历史演义小说, 英文全译本主要包括由外国学者邓罗、罗慕士翻译的两个版本 和中国学者虞苏美翻译的版本。目前国内学术界对《三国演义》英译本的理论研究以翻译学理论为主, 此外普 遍存在“襄罗贬邓”的趋势，更鲜有以多元系统论分析《三国演义》邓罗译本的研究。多元系统论以文本以外的 文化等多元系统丰富了翻译研究手段。本文以《三国演义》邓罗译本为研究对象，在多元系统论视阈下，探讨 中英文学所处的地位如何塑造译者翻译的意图，同时借助实例说明小说中不同元素的地位对翻译行为和选词 策略的影响，并指出多元系统论体现在邓罗译本的不足之处。

关键词: 多元系统论; 《三国演义》仍罗译本; 中国古典名著翻译; 翻译意图 


\section{1.引言}

以色列学者埃文·佐哈尔 (Even-Zohar) 早在 1977 年提出一个耳目一新的概念“多元系统” (polysystem), 用以描述某一特定文化里的各种文学系统的聚合, 从 诗这样“高级的”、或“经典的”形式，到“低级的”、或 “非经典的”形式。中国学者谢天振 (2003) 针对“多元 系统理论”作该理论综述的时候, 以“具有革新意义的 诗” 作为前者的例子, 而用儿童文学、通俗小说指代后 者。

多元系统论针对《三国演义》的翻译理论研究凤 毛麟角。有的学者认为该理论不合乎晚清时期的翻译 实际，但实际上《三国演义》并不在清末民初面世， 这只是邓罗译本出版时所处的阶段。另外亦有学者从 文化翻译策略归因方面解释《三国演义》罗慕士译本, 其中稍有涉及多元系统论 (贺显斌, 2003)。经相关学 者对比分析, 罗慕士译本多采用直译, 而邓罗译本以 省译为主, 更多体现多元系统论的特点。多元系统论 本身牵涉学科之多以及其本身对于翻译研究并不完 善, 而且我国关于邓罗译本的翻译研究明显少于罗慕 士译本。

本文主要研究两个问题: (1)《三国演义》在不同 文学多元系统中所处的地位如何塑造译者实施翻译 行为的意愿与动机? (2) 从实例出发, 小说中的各种 要素成分在《三国演义》系统中的不同地位如何影响 译者的翻译行为和选词策略?

\section{2.多元系统论概述}

多元系统理论的核心内容就是把各种由符号支 配的人类交际形式, 如语言、文学、经济、政治、意 识形态等, 视作一个系统而不是一个由各不相干的元 素组成的混合体。这个系统不是一个单一的系统, 而 是由不同成分的、开放的结构, 即是一个由若干个不 同的系统组成的多元系统。在这个多元系统里, 各个 系统“互相交叉, 部分重叠, 在同一时间内各有不同的 项目可供选择, 却又互相依存, 并作为一个有组织的 整体而运作 (张南峰, 2005)。”以下以本文研究对象 为例对多元系统论作一简单的阐释。在 《三国演义》 邓罗译本面世之际, 中国本土文学与外来引入中国的 翻译文学是一个多元系统中的两个系统, 而英语国家 文学与外来引入英语国家的中国文学也可以被视为 一个多元系统中的两个系统。与此同时, 在中国本土 文学这个多元系统中, 亦不乏儒学经典系统、唐诗宋 词系统、通俗小说系统等, 《三国演义》可以说是通俗 小说系统中的一员。在当今古典文化百花盛开、丰富 多彩的中国, 《三国演义》也可以被称之为一个多元 系统, 其文言版本、白话版本、青少版本等均为其子 系统。各个多元系统的地位在整个系统中并不平等, 于中心的、于边缘的, 不尽相同, 但是各系统的地位 并不是一经形成、不再改变的, 而是相互转变、相互 依存的。显然, 在多元系统论视角下, 在不同时期,
《三国演义》汉语本和英译本也各自处在不同的多元 系统中的不同位置。

埃文·佐哈尔 (1979) 特别指出了翻译文学在多元 系统论中的位置。他认为, 多元系统论将更多在往前 某一段时间被忽略或是被排斥的现象纳入翻译的研 究范围, 不仅翻译的社会文学地位取决于它在多元系 统内的位置, 翻译的实践也完全由此主导。由此可见, 多元系统论结合翻译和文化两者的研究, 将源语文本 和目的语文本的相互联系过程产生的文化语境、社会 条件、政治等许多因素结合起来, 为翻译理论研究开 拓了更广阔的视野（谢天振，2003）。

多元系统论也不乏批判之声。本文列出三处实例 既呈现多元系统论在《三国演义》邓罗译本中的指导 意义，也指出多元系统论的不足之处主要在于其单纯 考虑制约翻译策略选择的客观文化因素, 而忽视了作 为翻译主体的人的主观能动性 (张建青, 2010), 因而 邓罗译本中显现的不同处理手段不能完全反映多元 系统论指出的单纯因文化而影响的翻译行为。除了邓 罗在翻译《三国演义》时考虑中英两种文化在世界文 化系统中所处的地位，他自身受到中国文化多年的熏 陶所引起的主观文化判定对其译作也大有影响。

\section{3.多元系统论对《三国演义》邓罗译本的解读}

埃文·佐哈尔的多元系统论较为全面地分析了翻 译文学在译入语文学的多元系统的三种客观条件, 其 一是多元系统由于译入语本土文学发展处于“幼嫩” 状态而尚未定形，还有待确立；其二是译入语本土文 学处于“边缘”或“弱势”位置，甚至两者并存；其三是 译入语本土文学出现转折点、危机或文学真空（谢天 振，2003)。回溯到清末民初时，中国无论是在经济层 面还是政治层面都不如大部分西方国家，本土文学系 统在中国文学多元系统中可谓是并无过多立足之地, 相反国外引入的翻译文学系统则占据着该多元系统 的大部，中国古典文学在此阶段意欲“走出国门”实属 难上加难。对于英美国家而言, 该阶段的翻译文学 (外 文翻译成英文) 则与我国的情况恰恰相反，处于边缘 位置。即使英美本土作家, 尤其是汉学家, 对中国古 典文学作英译, 并以其本国读者为导向, 也未必能改 变此时多元系统的现状。

根据资料记载，邓罗早在 1880 年初访中国，凭 借其过人的语言天赋, 利用短短数年已熟识汉语并广 泛涉猎中国古典著作。五年以后，他开始接触中国作 品的翻译，有过两次翻译《三国演义》的经历，因而 产出两本译稿。第一译稿可谓“生不逢时”，毁于义和 团运动; 二稿可以说是“艰难玉成”于 1925 年出版问 世。从历史可知, 1925 年的中国处于半封建半殖民地 社会阶段，资本主义在民国政府的领导下如雨后春笋 般发展，广收博采、兼容并蓄的风气弥漫社会。显然， 从邓罗初至中国直至其译作“Romance of the Three Kingdoms”正式出版, 此时的中国现代文学恰处“细嫩” 状态。在当时中国的文学界, 英译中翻译文学和中国 
本土文学恰似“众星拱月”之景, 前者为“月”, 处于中 心，后者为“星”，处于边缘。邓罗的译作却是一部处 于边缘位置文学的英译作品, 他也因而开创了全译中 国经典名著的先河。但是, 若从多元系统论的现实角 度考虑, 这显然不是一个常规的举措, 处于边缘位置 的译入语文学在当时的历史背景下难以与英译中的 翻译文学匹敌。换一个角度考虑, “Romance of the Three Kingdoms”的受众对象主要是以英语为母语的 读者, 而英语国家本土文学在当地处于中心位置, 译 入语文学显然处于边缘, 处于半封建半殖民时期的中 国文学更是“人微言轻”。邓罗的长达 15 年艰苦劳作 完成的第一版英译本手稿被打着“扶清灭洋”旗号的 义和团运动爆发而灰飞烟灭, 但是在这种时代背景下 邓罗并没有放弃第二版英译本的创作。该译本的推出 涉及到多元系统论的关于中心与边缘位置分析的不 足。

埃文·佐哈尔认为, 在某些运动中, 一个项目 (元 素或功能) 可能从一个系统的边缘转移到同一个多元 系统中的相邻系统的边缘, 然后可能走进 (也可能走 不进) 后者的中心 (张南峰, 2005)。在邓罗译本出版 的时候, 在英语国家的多元系统中, 本土文学占据主 导, 翻译文学屈居其次, 但是邓罗译本一上市就受到 普罗大众的交口称誉, 深受追捧, 包括金陵大学第一 任校长 (A.J.Bowen) 在内的文人雅士都向邓罗表示祝 贺, 英国皇家亚洲学会中国北方分会杂志也提到了邓 罗译本 (陈甜, 2013)。邓罗译本尽管难以在当时英语 国家文学系统中走向中心, 也算得上是作为翻译文学 的逆袭。因此, 翻译文学在文学多元系统中并非永远 位居次席, 也可能逐渐走向中心。当然, 在邓罗译本 出版时期, 外国文学在中国甚至塑造了多元系统的中 心部分。

邓罗在翻译《三国演义》的时候, 中国文学系统 内部其实已经形成了多元系统, 当时儒学经典和唐诗 宋词处于该多元系统的中心地位, 通俗小说实则处于 边缘位置。这种多元系统在很大程度上也影响了西方 的汉学研究。在西方的中国文学翻译系统中, 仍然以 儒家作品和唐诗宋词的英译作品为核心, 通俗小说的 英译作品则鲜有出版。在这种历史背景下, 《三国演 义》邓罗译本还是成功面世。从邓罗的“Romance of the Three Nations”译作前言的内容可以得知其在当时文 化系统背景下坚持选择翻译《三国演义》的原因: 第 一, 邓罗认为《三国演义》是一部引人入胜的小说, 饱含东方色彩与元素, 以英语国家读者能够深入了解 原著为目的; 第二, 《三国演义》在当时已有不少东方 语译本, 却没有作为英语这一通用语言的译本。基于 以上两个原因, 作为英国汉学家的邓罗决心将《三国 演义》译成英语。《三国演义》在邓罗的笔下只是由于 其呈现出极大的文化内涵而译作英文, 而非主要因为 多元系统论所提到的译入语文学与翻译文学的文化 建构。由此可见, 中国通俗小说虽然在多个不同的多 元系统中都处于劣势, 仍然有译者进行翻译, 足以说 明“人的主观能动性”对于译者行为与策略的影响, 多
元系统论在此难以解释。正如根茨勒指出, 多元系统 论倾向于关注抽象的模式而非“社会现实中实际”加 诸文本和译者之上的各种制约因素; 重要的文学外部 因素远不在其分析视域（Munday，2016）。

佐哈尔将翻译文学在文学多元系统的位置延伸 至该位置对翻译的规范、行为模式、翻译策略等的影 响问题。佐哈尔 (1979) 认为, 当翻译文学处于文学 多元系统的边缘位置时, 译者的主要工作, 就是为外 国的文本, 找来最佳的现成二级模式, 结果是译本的 充分性不足。如果我们从英语国家的角度考虑多元系 统论, 显然本土文学置于中心, 翻译文学置于边沿。 尽管邓罗以为英语读者提供最真实的中国名著为目 的, 他的译作更多的是以英语国家本土文化为一级模 式, 从而与译入语文化搭配, 引申而成二级模式。但 是, 从实例可见, 我们也可以看到并不完全符合这一 看法, 这也是众多学者指出的关于多元系统的不足之 处。

除此之外, 亦有学者认为, 佐哈尔对文学系统状 态的描述有些粗粘, 对弱小、边缘的评价性陈述并不 明晰 (张建青, 2010)。根据邓罗的描述, 中国经典名 著从文化的层面并不弱小与边缘, 反而可能处于主导 位置, 但是在当时大部分不为英语国家的读者所知。 《三国演义》作为中国四大名著之一脍多人口, 邓罗 译本作为第一部全译本也是喜闻乐见, 单纯从 《三国 演义》在中国文学多元系统中的地位绝对是位高权重。 即使从世界文学系统来探讨, 中国文学也不应被列为 边缘的位置, 更大程度上只是受制于当时中国的经济、 政治等地位。如果从经济、政治的多元系统的角度, 认为《三国演义》的译作属于边缘地位, 无可厚非; 那么从文化、文学的多元系统的角度, 则难被信服。 可见，相关学者对于多元系统论“弱小、边缘”的界定 过于模糊的评价在《三国演义》邓罗译本中可以体现。

因此, 《三国演义》邓罗译本在中国处于内忧外患 严峻态势的年代诞生，不能单纯地归因于中国文化、 文学系统与西方文化、文学系统在多元系统中的地位 关系与角力权衡, 而译者对于原著的个人偏好以及译 者本人受原著所处文化的影响也在一定程度上成为 译作应运而生的原因。译者对翻译策略的选取与实施, 一方面取决于源语和目的语的文化建构, 另一方面决 定于译者对两者的认同感与偏好感, 前者是在多元系 统论视角下的客观因素, 后者则是该理论没有过多阐 释的主观因素。

\section{4. 《三国演义》邓罗译本的举例阐释}

例一: 幽州太守刘焉, 乃江夏竟陵人氏，汉鲁恭 王之后也。

译为: The Imperial Protector of Youzhou was Liu Yan, a scion of the Imperial House.

对于这一句关于“刘焉”的介绍, 译本明显省略了 “乃江夏竟陵人氏”, 即省略了其祖籍和出身。在中国 
传统文化中, 祖先崇拜的观念十分突出, 祖籍的追溯 和认定对于每一个家庭都十分重要。但是在西方文化, “祖籍”这一概念并不存在, 西方人看重的是“血统”, 尤其是英国人所说的“皇室血统”。因此，根据多元系 统论, 译者关注的是译入语文化读者对语义的接受, 西方本土文化关注的是“刘焉”皇室血统, 而非其祖籍 和出身, 在这一点上邓罗译本的语义传递足矣。

“太守”是秦汉时期对郡守的尊称，该官衔为郡最 高行政长官。当时的郡相当于现在的市, 郡守类似于 市长。“imperial”的英英释义为“belonging or relating to an empire or the person or country that rules it", 看似比 郡的范畴大得多。在古代, 朝发夕至、缓不济急较为 常见, 因此作为一郡的最高行政长官的太守往往大权 独揽, 形成割据势力。译者用了“imperial protector”, 一方面为了更大程度地反应中国古典文化的隐喻, 另 一方面通过符合英语读者文化认知的词汇, 达到其翻 译的目的。反之, 译者对“皇室后裔”的处理, 则用了 一个英释为“a young member of a family, especially a famous or important one”的单词“scion”, 因为在英国, 皇室成员十分尊贵, 译者没有以“royal descendant”进 行处理, 意味着没有过多给一部在英语国家出于边缘 文化的巨著的人物赋予过高地位的意图。

正如上文分析, 《三国演义》著作本身可视为一个 系统, 著作里的每一个人物可以视为各自独立而共同 为著作服务的元素。这一角度也可以解释此处省略信 息的原因, “刘焉”并非本书的主角, 处于这一多元系 统的边缘位置, 在人物元素纷繁复杂的《三国演义》 系统中出现频率少, 过多的介绍会引起喧宾夺主之嫌。 相反, “刘备”作为《三国演义》的主要人物, 处于这 一系统的中心位置, 对此人物的介绍相对详尽亦不为 过。

例二: 中山靖王刘胜之后, 汉景帝阁下玄孙, 姓 刘, 名备, 字玄德。

译为: He was a descendant of Prince Sheng of Zhongshan whose father was the Emperor Jing, the fourth emperor of Han Dynasty. His name was Liu Bei.

该句关联到小说重要人物出身, 而“中山”一词直 接以音译的形式出现在译文, 可见其对“刘备”祖籍的 强调。同时, 译者并未对该词加注, 虽不符合英语国 家文化为主导的多元系统论的要求, 但是以该著作为 多元系统的角度, 译者突破了译入语文化的限制和约 束, 有助于译文最大程度地接近原文的“充分性”。中 国古人除了用父母所予姓名外, 亦用“表字”, 出于所 生或所处地名、历史典故、或是本身姓名引申之别称。 以“表字”称呼其人, 既是表尊敬, 也是传亲切。在中 国传统文化中, “表字”是不可或缺的, 反映出民族独 特性。译者在此处只给出姓名之音译, 未提“表字”, 对英语读者而言较为友好, 无需处理过多人物姓名, 而是着重于故事情节。

例三: 其人曰: “某姓张, 名飞, 字翼德。世居派 郡, 颇有庄田, 卖酒屠猪, 专好结交天下豪杰。
译为: “Zhang Fei is my name," replied the stranger. "I live near here where I have a farm; and I am a wine seller and a butcher as well; and I like to become acquainted with worthy humans."

“豪杰”一词，意为才智勇力出众的人，多现中国 古代武侠小说, 正如“江湖”一词并不容易翻译。译者 以“worthy people”释之, 虽不能尽显“骁勇谋略双全” 的原意, 也足以反映这类人德高望重之地位, 易被英 语读者接纳。

“字翼德”与“溷郡”已省略, 从微观的角度, “字翼 德”与“涿郡”在书中都属于边缘元素, “张翼德”虽《三 国演义》三国演义中较为主要的任务人物, 但正如“字 玄德”的省略操作, 邓罗全书翻译均省去“表字”, 而书 中武将之多，逐一描述其家乡难免会给英语读者造成 一定的阅读困难, 尤其是对于以汉语拼音音译而成的 中国地名的记忆, 甚至可以说, 本族语的读者也未必 可以清楚地理清书中复杂的时地人事。从宏观的角度, 两者都难以在英语文化语境中找到相应之物, “表字” 与“地名”的省略, 看上去像是“削足适履”, 将原作套 入目的语文学的既有模式, 但是这也是依据文化关系 现状而决定的。

有学者则认为是此类省略是源于邓罗不能透彻 理解源语文化内涵, 其指出邓罗的翻译, 只能称之重 述, 而非翻译。但是从多元系统论的角度, 在英语国 家文化占据主流的现实背景下, 邓罗对于这些内容选 择直接回避, 虽然不能完全传递出中国传统文化, 但 是多元系统论对翻译文学的阐述形成的看待“充分性” 的新角度确认为是时代所造就的合理性。这种情况也 好比古罗马人对希腊典籍的翻译。

因此，译者既承载着传递中国古典文化的“使命”, 也需要考虑英语国家读者的接受程度, 在两者之间的 权衡中受到多元系统论考虑的客观文化因素以及译 者的主观能动性的影响, 从而对于处理原著中的不同 组成部分和要素, 存在着不同的策略取向, 进一步形 成其选词策略成分。从实例中可见, “省译”是邓罗译 本翻译行为中的很大组成部分, 如果从多元系统论视 角下看待 “充分性”, 如此行为自然有其合理之处, 最 终实现译者所意向的将中国古典文化带给英语读者 的目的。

\section{5.结论}

邓罗在翻译《三国演义》之前早已经在中国生活 多年, 较为熟悉地了解中国文化, 译者对《三国演义》 英译的意愿与动机、翻译行为以及选词策略不仅受到 译出语和译入语文学在世界文学多元系统中的地位 的影响, 还受到译者自身对中国古典文化的认同度以 及推动中国古典文化走进英语国家文化系统的积极 性的主观影响。自古以来, 国内外研究对《三国演义》 邓罗译本褒贬不一。在多元系统翻译理论视角下, 邓 罗译本在传承中国文化、迎合英语读者、合理调整省 译等方面有一定的建树。邓罗译本符合地道英语, 以 
英语国家文化为导向的同时, 也尽可能保留了中国古 典文化的风格韵味。邓罗作为著名汉学家, 翻译《三 国演义》的初衷是将中国的历史与文化传递给西方人。 追本溯源, 《三国演义》邓罗译本面世之日亦是中国 文化在世界文化系统中处于劣势之时, 译者对二次译 作的坚持是其对中国文化满腔热诚的真实写照, 这是 多元系统论忽视“人的主观能动性”的最好诠释。

\section{REFERENCES}

[1] Brewitt-Taylor, C. H. (1925) Translation, San Kuo, or Romance of the Three Kingdoms. An English version of San Kuo Chih Yen-i. 2 Vols. by Lo Kuanchung. Kelly and Walsh, Shanghai.

[2] Chen, T. (2013) Re-evaluation of Brewitt-Taylor's translation of San Guo Yan Yi. Academic Journal of Zhongzhou, (9): 162-165.

[3] Even-Zohar, I. (1979) Polysystem Theory. Poetics Today, (1): 287-310.

[4] Even-Zohar, I. (2002) Polysystem Theory (N. Zhang, Trans.). Chinese Translators Journal, (4).

[5] He, X. (2003) New attribution for cultural translation strategies. Journal of Tianjin Foreign Studies University, (6): 1-6.

[6] Munday, J. (2016) Systems theories. In: Jeremy, M. (Ed.), Introducing Translation Studies: Theories and Applications ( $4^{\text {th }}$ ed.). Routledge, London. pp. 169196.

[7] Xie, T. (2003) Polysystem Theory: A new approach to translation studies. Journal of Foreign Languages, (4): 59-66.

[8] Zhang, J. (2010) On Even-Zohar's Polysystem Theory in translation. Foreign Language Education, 31(3): 110-113.

[9] Zhang, N. (2005) The "nationality" of translated literature from a polysystemic perspective. Journal of Foreign Languages, (5): 54-60. 